\title{
Eficiência dos protocolos de tratamento em uma e duas fases da má oclusão de Classe II, divisão 1*
}

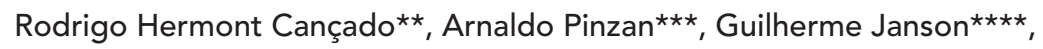

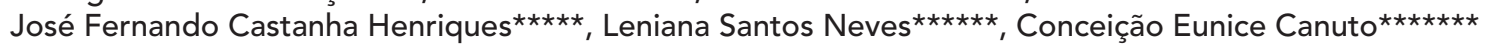

\section{Resumo}

Objetivo: o objetivo deste estudo retrospectivo foi comparar os resultados oclusais e a eficiência dos protocolos de tratamento em uma e duas fases da má oclusão de Classe II, divisão 1. A eficiência do tratamento foi definida como um melhor resultado oclusal em um menor tempo de tratamento. Métodos: uma amostra de 139 pacientes com má oclusão de Classe II, divisão 1, foi dividida em dois grupos, de acordo com o protocolo de tratamento instituído para a correção da má oclusão: grupo 1 - constituído por 78 pacientes tratados com o protocolo de tratamento em uma fase, com idades médias inicial e final de 12,51 $( \pm 1,28)$ e 14,68 $( \pm 1,49)$ anos, respectivamente; e grupo 2 - constituído por 61 pacientes tratados com o protocolo de tratamento em duas fases, com idades médias inicial e final de $11,21( \pm 1,21)$ e 14,70 $( \pm 1,55)$ anos, respectivamente. Telerradiografias em norma lateral foram obtidas ao início do tratamento, para avaliar a compatibilidade cefalométrica dos grupos. Os resultados oclusais foram avaliados nos modelos de gesso dos pacientes nas fases inicial e final utilizando o índice IPT (Índice de Prioridade de Tratamento). Aplicou-se o teste do quiquadrado para avaliar a compatibilidade dos grupos quanto à severidade da relação ântero-posterior dos arcos dentários e à distribuição por gêneros. As variáveis diretamente relacionadas aos resultados oclusais foram comparadas por meio do teste $t$ independente. Finalmente, uma análise de regressão linear múltipla foi realizada, a fim de verificar a influência de diversas variáveis no tempo de tratamento. Resultados: verificou-se que resultados oclusais semelhantes são obtidos com os protocolos de tratamento em uma e duas fases. No entanto, o tempo de tratamento foi significantemente menor no grupo tratado em uma fase. Conclusão: baseado nos resultados desse trabalho pode-se concluir que o tratamento da má oclusão de Classe II, divisão 1, é mais eficiente quando realizado com o protocolo de tratamento em uma fase.

Palavras-chave: Má oclusão Classe II de Angle. Resultado de tratamento. Aparelhos ativadores.

* Resumo da tese de doutorado apresentada à Faculdade de Odontologia de Bauru-USP, 2005.

* Mestre e doutor em Ortodontia pela Faculdade de Odontologia de Bauru - USP. Professor adjunto da Unidade de Ensino Superior Ingá (UNINGÁ). Professor do curso de Especialização em Ortodontia da Universidade Federal dos Vales do Jequitinhonha e Mucuri (UFVJM).

*** Professor associado do departamento de Odontopediatria, Ortodontia e Saúde Coletiva da Faculdade de Odontologia de Bauru - USP.

**** Professor titular do departamento de Odontopediatria, Ortodontia e Saúde Coletiva da Faculdade de Odontologia de Bauru - USP. Coordenador do curso de pós-graduação ao nível de mestrado em Ortodontia pela Faculdade de Odontologia de Bauru - USP. Membro do "Royal College of Dentists of Canada".

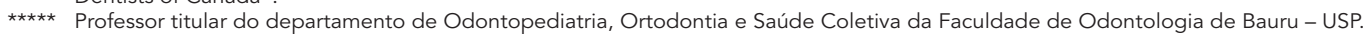

$\star \star \star \star \star \star$ Mestre e doutora em Ortodontia pela Faculdade de Odontologia de Bauru - USP. Professora do curso de Especialização em Ortodontia da Universidade Federal dos Vales do Jequitinhonha e Mucuri (UFVJM).

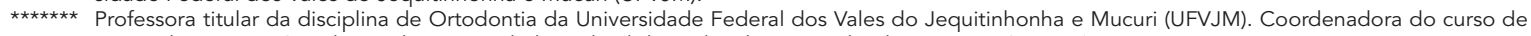
especialização em Ortodontia da Universidade Federal dos Vales do Jequitinhonha e Mucuri (UFVJM). 


\section{INTRODUÇÃO}

Para o tratamento da má oclusão de Classe II há uma miríade de aparelhos, uma vez que esta má oclusão desperta um grande interesse clínico e científico, por constituir uma porcentagem significativa dos casos na clínica ortodôntica ${ }^{11,12,18,29}$. Essa diversidade de modalidades terapêuticas freqüentemente gera dúvidas e indecisões quanto à escolha da abordagem que melhor se aplica a cada caso.

Dentre as diversas modalidades de tratamento disponíveis para a má oclusão de Classe II, os aparelhos fixos e os aparelhos ortopédicos funcionais estão entre os recursos terapêuticos de maior efetividade ${ }^{12,29}$. No entanto, a época ideal para iniciar o tratamento da má oclusão de Classe II é um tema ainda hoje bastante controverso.

O protocolo de tratamento em duas fases preconiza o início do tratamento durante a préadolescência e dentadura mista com a utilização dos aparelhos ortopédicos funcionais e uma segunda fase na adolescência, após a irrupção dos dentes permanentes, em que o tratamento é complementado com aparelhos fixos ${ }^{9}$. O protocolo de tratamento em uma fase consiste na realização do tratamento ortodôntico com aparelhagem fixa em uma idade mais avançada, uma vez que o paciente deverá apresentar todos os dentes permanentes irrompidos na cavidade bucal. De acordo com esse protocolo, a fase ortopédica com aparelhos funcionais é eliminada, uma vez que a melhoria do padrão esquelético do paciente também é conseguida com a utilização de aparelhos extrabucais (IHG ou KHG) ${ }^{10,31}$ associados ou não a elásticos de Classe II $^{24,26}$ e, por conseguinte, sem a utilização dos aparelhos ortopédicos funcionais ${ }^{23}$.

Alguns autores relatam que os melhores resultados terapêuticos e uma maior estabilidade são obtidos quando o tratamento da Classe II é realizado em duas fases ${ }^{2,9}$. No entanto, essa assertiva é motivo de constante controvérsia, uma vez que a influência da fase ortopédica nos resultados clíni$\cos$ finais é praticamente inexistente $e^{21,22,33}$.
A escolha por um determinado protocolo terapêutico está na dependência de inúmeros fatores, como efetividade, fatores psicológicos, financeiros, riscos de dano aos tecidos dentários e ao periodonto de sustentação, complexidade do tratamento, duração, estabilidade e aceitação do método pelo paciente ${ }^{27,28}$. Esse último fator está diretamente relacionado com a colaboração conseguida durante o tratamento ${ }^{19}$. Por isso, a opção por um determinado protocolo para o tratamento da má oclusão de Classe II requer que o profissional conheça profundamente as principais indicações e contra-indicações de cada protocolo, assim como a eficiência de cada um deles. Como são amplamente utilizados no tratamento da Classe II, julgou-se oportuno comparar a eficiência dos protocolos em uma e duas fases no tratamento da Classe II. As informações obtidas auxiliarão o clínico no planejamento do tratamento dessa má oclusão, considerando-se principalmente a época ideal para tratamento, a necessidade de cooperação do paciente e o tempo total de tratamento.

\section{MATERIAL E MÉTODOS Material}

Procedeu-se à seleção da amostra, de caráter retrospectivo, a partir do arquivo da Disciplina de Ortodontia da Faculdade de Odontologia de Bauru da Universidade de São Paulo (FOB-USP). A amostra, constituída de 278 pares de modelos de gesso, obtidos no início e no final do tratamento ortodôntico, e 139 telerradiografias em norma lateral de 139 pacientes, obtidas ao início do tratamento, foi dividida em dois grupos:

- Grupo 1: composto por 78 pacientes, sendo 41 do gênero masculino e 37 do gênero feminino, com idade inicial média de 12,51 anos (d.p. $=1,28$, mínimo $=9$,08 e máximo $=16,03$ ), que apresentavam, ao início do tratamento, má oclusão de Classe II, divisão 1, os quais foram tratados em uma fase com aparelhos fixos da técnica Edgewise, nos arcos superior e inferior, por um período médio de 2,17 anos (d.p. $=0,66$, mínimo $=$ 0,99 e máximo $=4,60$ ), finalizando o tratamento 
Tabela 1 - Médias, desvios-padrão, valores mínimo e máximo das idades iniciais e finais e do tempo total de tratamento nos dois grupos estudados.

\begin{tabular}{|c|c|c|c|c|c|c|c|c|}
\hline & \multicolumn{4}{|c|}{ grupo $1(n=78)$} & \multicolumn{4}{|c|}{ grupo $2(n=61)$} \\
\hline & média & d.p. & mín. & máx. & média & d.p. & mín. & máx. \\
\hline idade inicial & 12,51 & 1,28 & 9,08 & 16,03 & 11,21 & 1,21 & 8,00 & 14,00 \\
\hline idade final & 14,68 & 1,49 & 10,75 & 18,56 & 14,70 & 1,55 & 11,99 & 19,49 \\
\hline tempo trat. & 2,17 & 0,66 & 0,99 & 4,60 & 3,49 & 1,56 & 1,45 & 7,02 \\
\hline
\end{tabular}

com idade final média de 14,68 anos (d.p. = 1,49, mínimo $=10,75$ e máximo $=18,56)($ Tab. 1$)$.

- Grupo 2: composto por 61 pacientes, sendo 30 do gênero masculino e 31 do gênero feminino, com idade inicial média de 11,21 anos (d.p. $=1,21$, mínimo $=8,00$ e máximo $=14,00$ ), que apresentavam, ao início do tratamento, má oclusão de Classe II, divisão 1, os quais foram tratados inicialmente com um aparelho ortopédico funcional (ativador associado ao aparelho extrabucal ou Bionator de Balters) e, ao término dessa primeira fase, o tratamento foi complementado com uma segunda fase de aparelhos fixos da técnica Edgewise nos arcos superior e inferior. O tempo médio de tratamento foi de 3,49 anos (d.p. = 1,56, mínimo $=1,45$ e máximo $=7,02$ ) e a idade final média dos pacientes foi de 14,70 anos (d.p. = 1,55, mínimo $=11,99$ e máximo $=19,49)($ Tab. 1$)$.

Os seguintes critérios foram utilizados para a seleção da amostra:

- os pacientes dos dois grupos estudados deveriam apresentar, ao início do tratamento, uma má oclusão de Classe II, divisão 1, verificada nos modelos de gesso;

- o tratamento de todos os pacientes foi conduzido sem a extração de dentes permanentes;

- o tratamento dos pacientes foi realizado por alunos dos cursos de pós-graduação, lato e stricto sensu, e Aperfeiçoamento da Disciplina de Ortodontia da Faculdade de Odontologia de Bauru;

- não apresentavam agenesias ou perdas de dentes permanentes;

- não apresentavam dentes supranumerários;
- não se submeteram a qualquer tipo de tratamento ortodôntico previamente à utilização destes aparelhos.

É importante ressaltar que não foi realizada nenhuma avaliação cefalométrica a fim de se obter um diagnóstico diferencial para a inclusão e/ou exclusão dos pacientes em um determinado grupo em função de valores numéricos ou análise crítica dos dados. Assim sendo, todos os pacientes que satisfizessem os critérios de seleção acima citados, independente da qualidade dos resultados oclusais ao término do tratamento, foram incluídos na amostra.

Os pacientes do grupo 2 foram tratados com aparelhos distintos durante a fase ortopédica. Considerando a possibilidade desta ocorrência influenciar os resultados, o grupo 2 foi subdividido em subgrupos A e B. Dessa forma, o subgrupo 2A foi composto de 30 pacientes que foram tratados na fase ortopédica com o Ativador associado ao aparelho extrabucal e o subgrupo 2B foi composto de 31 pacientes tratados com o Bionator de Balters na primeira fase do tratamento.

\section{Descrição dos aparelhos empregados} Aparelho fixo (Fig. 1, 2, 3)

Os aparelhos fixos da técnica Edgewise simplificada com slot 0,022" x 0,030" foram utilizados nos dois grupos estudados, sendo que no grupo 1 foi associado ao aparelho extrabucal (IHG ou KHG) e/ou elásticos de Classe II para a correção da má oclusão de Classe II ${ }^{10,24,26,31}$, ao passo que no grupo 2 esse aparelho foi utilizado após o tratamento 
com um aparelho ortopédico funcional (Ativador ou Bionator de Balters). De um modo geral, os pacientes utilizaram, associado ao aparelho fixo, um aparelho extrabucal (KHG ou IHG) para a correção da relação molar de Classe II (pacientes do grupo 1) ou como forma de contenção da correção do relacionamento ântero-posterior entre os arcos dentários após o tratamento ortopédico (pacientes do grupo 2). Na grande maioria dos 61 pacientes do grupo 2, foi obtida a correção da relação molar de Classe 2 na $1^{\text {a }}$ fase do tratamento (fase ortopédica) e, nesses casos, durante a $2^{a}$ fase do tratamento (fase ortodôntica), a ancoragem extrabucal foi utilizada por aproximadamente 10 horas como contenção da correção da relação molar de Classe II. Evidentemente, nem todos os pacientes do grupo 2 obtiveram a correção da relação molar já na $1^{\text {a }}$ fase do tratamento e, nesses casos, durante a $2^{\text {a }}$ fase, a ancoragem extrabucal (IHG ou KHG) continuou sendo utilizada não como forma de contenção, mas sim como forma de correção da
Classe II que não foi totalmente corrigida na primeira fase do tratamento. Além disso, nos estágios finais do tratamento, freqüentemente utilizava-se elásticos intermaxilares de Classe II. Ao término do tratamento, procedia-se à instalação de uma placa de Hawley no arco superior e colagem do $3 \times 3$ no arco inferior.

\section{Ativador associado ao aparelho} extrabucal (Fig. 4, 5)

O Ativador associado ao aparelho extrabucal (IHG), utilizado na Disciplina de Ortodontia da Faculdade de Odontologia de Bauru-USP, apresenta algumas modificações quando comparado ao Ativador original de Andresen. O Ativador utilizado nessa pesquisa possui estruturas de acrílico superior e inferior unidas na região oclusal, com um parafuso expansor na parte superior, grampos de retenção do tipo Adams, um arco vestibular e um aparelho extrabucal cujo braço interno pode ser colocado diretamente no acrílico ou inserido

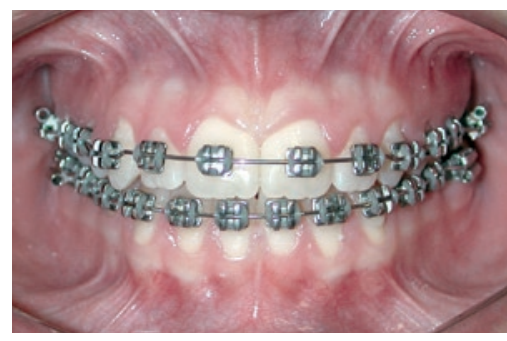

FIGURA 1 - Aparelho fixo.

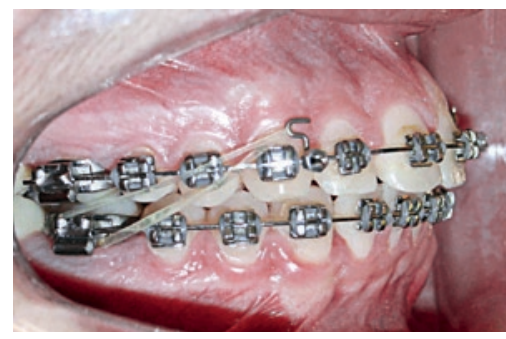

FIGURA 2 - Aparelho fixo associado aos elásticos de Classe II.

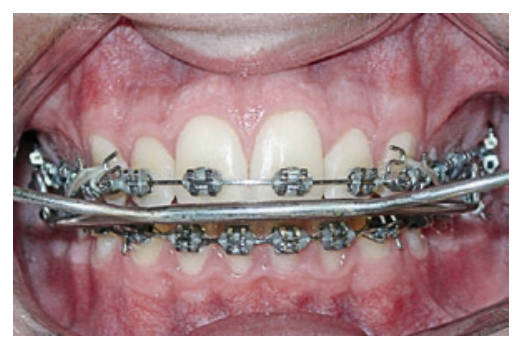

FIGURA 3 - Aparelho fixo associado aos elásticos de Classe II e ao aparelho extrabucal.

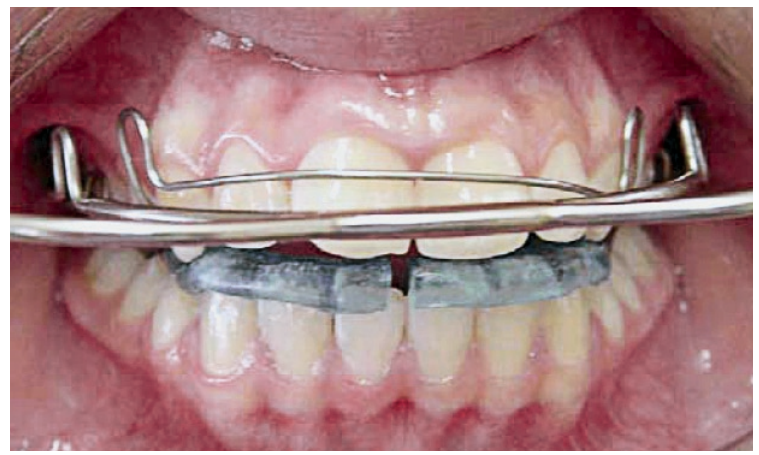

FIGURA 4, 5 - Ativador associado ao aparelho extrabucal (IHG).

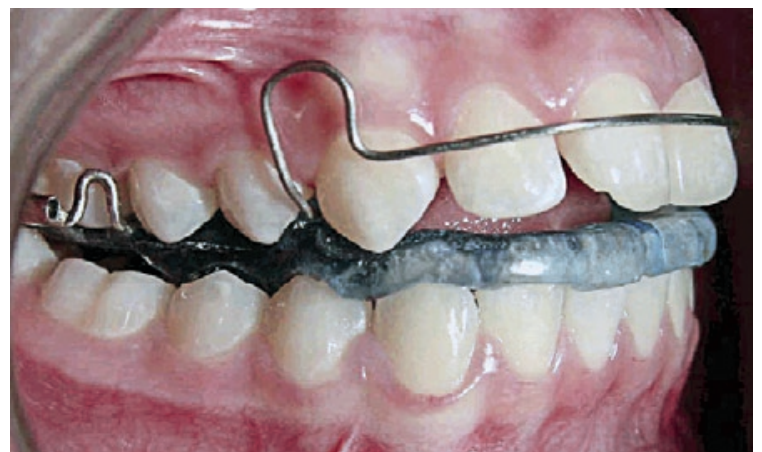


em tubos que são previamente soldados aos grampos de Adams. O acrílico superior recobre parcialmente o palato duro e as superfícies oclusais dos dentes póstero-superiores. Também apresenta um recorte na região da sutura palatina mediana, no qual é adaptado o parafuso expansor para evitar o desenvolvimento de uma mordida cruzada posterior durante o tratamento. $\mathrm{O}$ arco vestibular é inserido no acrílico por distal dos caninos superiores e toca a superfície vestibular dos incisivos superiores. Esse componente, além de auxiliar na retenção e estabilidade do aparelho, também proporciona um razoável controle da inclinação e do alinhamento dos dentes ântero-superiores. O componente de acrílico inferior recobre as superfícies oclusais dos dentes póstero-inferiores. Como via de regra, as bordas incisais dos incisivos inferiores também são recobertas para restringir o desenvolvimento vertical desses dentes, servir de referência para a protrusão mandibular quando do desgaste do acrílico na região posterior e diminuir a tendência à protrusão dos incisivos inferiores. O componente de acrílico inferior também possui extensões, recobrindo as superfícies linguais dos dentes inferiores e parte dos seus processos alveolares, sendo recortado ao nível dos incisivos centrais inferiores. Essas extensões de acrílico, associadas ao recobrimento das superfícies oclusais dos dentes póstero-superiores e inferiores, guiam a mandíbula para a posição desejada nos sentidos ântero-posterior e vertical.

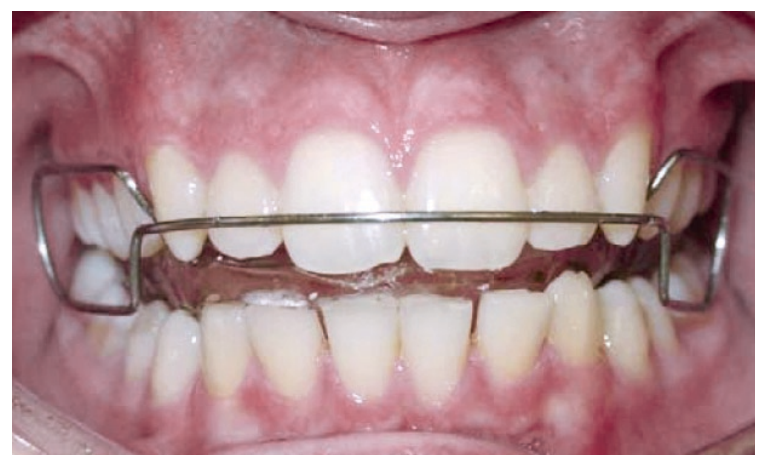

FIGURA 6, 7 - Bionator de Balters.
O aparelho extrabucal (IHG) possui uma angulação de aproximadamente $45^{\circ}$ em relação ao plano oclusal, a fim de permitir que as forças extrabucais passem o mais próximo possível do centro de resistência da maxila, mantendo o Ativador em posição. Elásticos 0,5”, trocados em média a cada três dias, forneciam uma força extrabucal em torno de 350 a $600 \mathrm{~g}$. As principais finalidades da associação do AEB (IHG) ao Ativador são: 1) restringir o deslocamento para anterior da maxila e 2) controlar os efeitos verticais desfavoráveis que o aparelho funcional pode acarretar.

Recomendava-se que os pacientes utilizassem o Ativador associado ao aparelho extrabucal (IHG) por um período de aproximadamente $20 \mathrm{~h} /$ dia.

\section{Bionator de Balters (Fig. 6, 7)}

O Bionator de Balters utilizado foi construído de acordo com o desenho original de Balters e adaptado por Ascher ${ }^{1}$. O Bionator apresenta, na sua constituição, quatro componentes básicos: uma estrutura de acrílico, um arco palatino, um arco vestibular e dobras do bucinador. De acordo com Balters, o objetivo do arco palatino é estimular a parte distal da língua. O objetivo do arco vestibular, de acordo com Balters, é criar uma suave pressão negativa entre os incisivos superiores e a mucosa dos lábios, o que contribuirá para manter esses dentes mais verticalizados. As dobras do bucinador têm dois objetivos no tratamento: 1) manter afastados os tecidos moles das bochechas e 2) deslocar

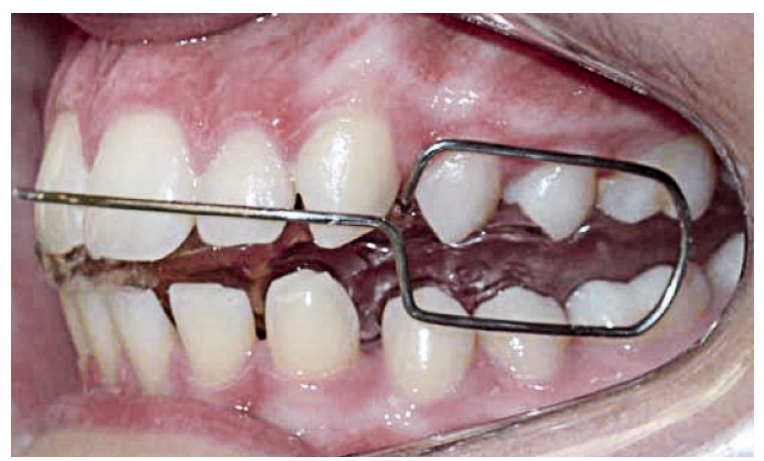


as bochechas lateralmente, aumentando o espaço bucal em virtude do posicionamento para frente da mandíbula, relaxando a musculatura, enquanto o arco vestibular a mantém afastada da mucosa alveolar. Algumas estruturas desse aparelho diferiram da forma original proposta por Balters. A porção ântero-inferior de acrílico recobria a borda incisal dos incisivos superiores e inferiores, diminuindo o desenvolvimento vertical e a protrusão desses dentes ${ }^{30}$. O arco vestibular construído originalmente por Balters para guiar ou facilitar o selamento labial toca a superfície vestibular dos incisivos superiores, funcionando como elemento ativo durante o tratamento ${ }^{30}$.

Técnica de obtenção da mordida construtiva para o Ativador e o Bionator de Balters

Para a obtenção da mordida construtiva, foi utilizado um rolete de lâmina de cera 7 com as seguintes finalidades: 1) fornecer uma orientação para a construção dos aparelhos ortopédicos funcionais e 2) determinar o grau de ativação desses aparelhos. O correto posicionamento da mandíbula nos três planos do espaço - horizontal, vertical e transversal - é condição sine qua non para o sucesso de um aparelho funcional. No sentido ântero-posterior, realizou-se um avanço máximo de $7 \mathrm{~mm}$, até que os incisivos superiores e inferiores ficassem em uma relação de topo-a-topo. Naqueles casos em que o paciente apresentava um trepasse horizontal maior que $7 \mathrm{~mm}$, houve a necessidade da realização de um outro avanço mandibular. A relação de molares e caninos serviu como um referencial para o posicionamento mandibular no sentido ântero-posterior. No sentido vertical, o fator determinante foi a quantidade de sobremordida inicial do paciente e, de uma maneira geral, obteve-se uma abertura entre os incisivos superiores e inferiores de 2 a $3 \mathrm{~mm}$. No sentido transversal, avaliou-se o relacionamento entre as linhas médias dentárias posicionando a mandíbula do paciente em relação cêntrica. Caso houvesse uma coincidência entre as linhas médias dentárias, a mordida construtiva também era obtida com as linhas médias coincidentes. Se, durante a avaliação em relação cêntrica, não houvesse uma coincidência entre as linhas médias, era necessário determinar se este desvio era de natureza dentária ou esquelética. Se o desvio fosse de natureza dentária, obtinha-se a mordida construtiva sem corrigir esse desvio, porém, se o desvio fosse de natureza esquelética, a mordida construtiva era obtida corrigindo-se esse desvio entre as linhas médias dentárias.

\section{Métodos}

Modelos de gesso

O índice oclusal IPT (Índice de Prioridade de Tratamento)

O Índice IPT foi utilizado para a avaliação dos resultados oclusais e das relações intra e interarcos. Este índice foi escolhido, dentre vários outros, por ser cientificamente confiável e apresentar elevada reprodutibilidade ${ }^{14}$. Além disso, sua utilização possibilita: 1) avaliar a severidade da má oclusão previamente ao tratamento ortodôntico; 2) avaliar a melhora da má oclusão após o tratamento ortodôntico; 3) comparar a melhora da má oclusão entre os dois protocolos de tratamento selecionados; e 4) comparar a eficiência do tratamento entre estes dois protocolos, alcançando, assim, o objetivo principal dessa pesquisa.

\section{Obtenção do Índice IPT ${ }^{14}$}

A obtenção do IPT foi realizada a partir de uma tabela (Tab. 2) onde estão descritas as principais características da má oclusão a serem avaliadas, bem como os parâmetros necessários para essa avaliação.

1) Relação molar

O primeiro passo para a obtenção desse índice foi a classificação da relação molar que estabelece tanto a coluna da tabela a ser utilizada quanto a constante numérica a ser adicionada ao valor final do índice.

2) Sobressaliência

Uma vez definida a coluna apropriada ao tipo 
Tabela 2 - Componentes do índice IPT com seus respectivos escores ${ }^{14}$.

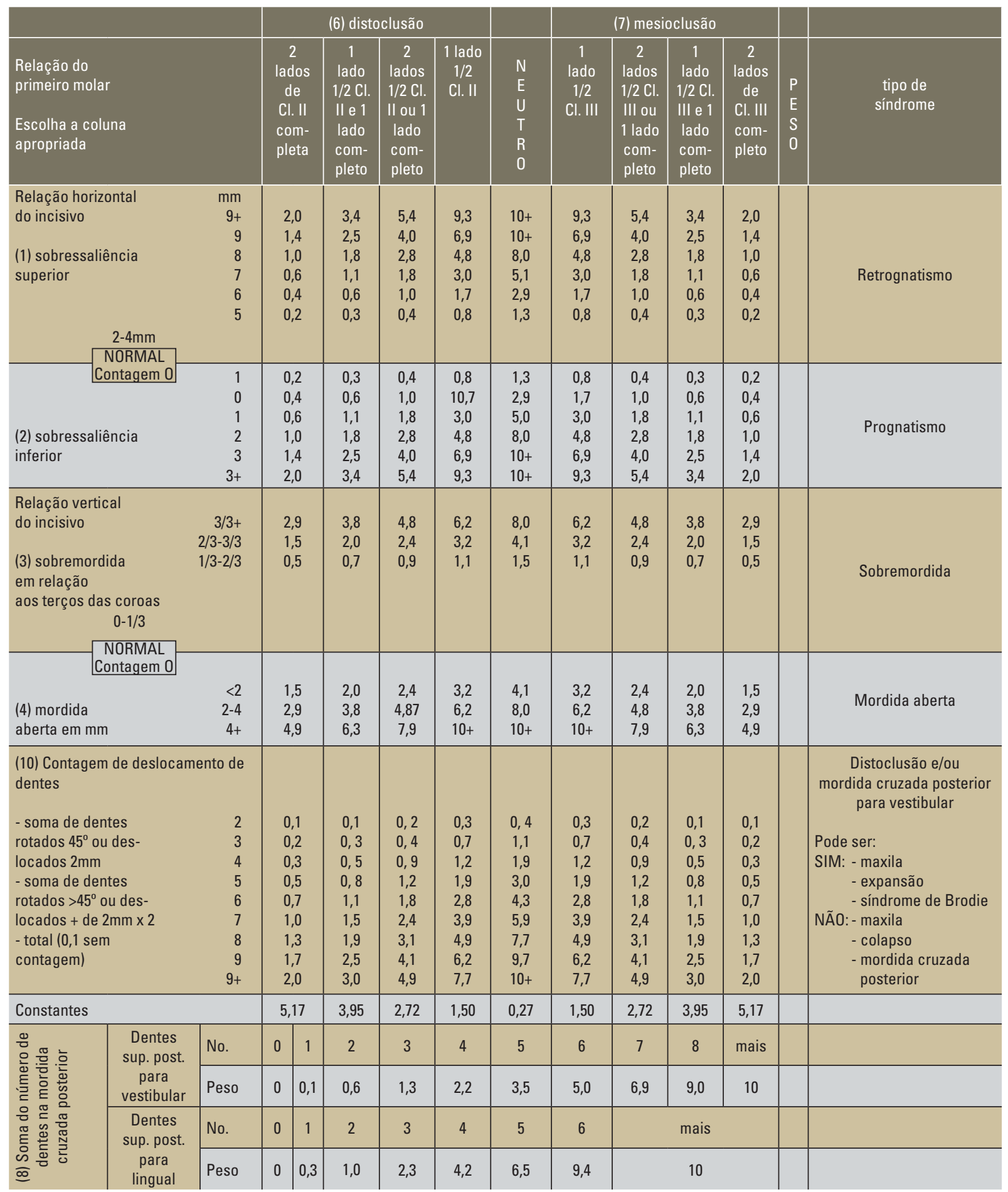

* A soma dos escores é o Índice de Prioridade de Tratamento. 
de má oclusão, o próximo passo foi realizar a mensuração, em milímetros, da quantidade de sobressaliência, tomando-se como referência a borda incisal dos incisivos superiores e a face vestibular dos incisivos inferiores. Para a mensuração da sobressaliência foi utilizada uma régua milimetrada. A partir do valor dessa medida obteve-se na tabela 2 o escore correspondente.

\section{3) Sobremordida}

A avaliação da sobremordida foi realizada tomando-se como referência a quantidade de terços da coroa dos incisivos inferiores que se encontravam encobertos pelos incisivos superiores, obtendo-se na tabela 2 o valor do escore correspondente.

4) Apinhamento e/ou rotação

A presença de apinhamentos e/ou rotações foi quantificada a partir da contagem do número de dentes que se encontravam deslocados ou girados em relação às suas posições ideais. Quando os dentes apresentavam uma giroversão em torno de $45^{\circ}$ ou se encontravam deslocados até $2 \mathrm{~mm}$ da sua posição ideal, os escores foram obtidos diretamente da tabela 2. Porém, quando os dentes apresentavam uma rotação maior que $45^{\circ}$ ou se encontravam deslocados mais que $2 \mathrm{~mm}$ em relação à sua posição ideal, o número de dentes com essas características foi multiplicado por dois.

5) Mordida cruzada

A contagem do número de dentes posteriores cruzados por vestibular (síndrome de Brodie) ou por lingual foi o critério utilizado para determinar na tabela 2 o escore atribuído a esse componente.

Após a obtenção dos escores para todos os componentes do índice IPT, realizou-se o somatório de todos esses escores adicionando-se ainda uma constante referente à relação molar inicialmente avaliada, portanto:

[IPT $=$ sobressaliência + sobremordida + apinhamento e/ou rotação + mordida cruzada + constante (relação molar)]

A diferença entre os valores inicial e final do índice IPT foi utilizada para se obter, em números absolutos, a quantidade de alteração do índice oclusal ocorrida com o tratamento. Além disso, foi também calculada a porcentagem de redução do índice IPT, utilizando-se a seguinte fórmula matemática:

Porcentagem de re- $=$ IPT inicial - IPT final dução do índice IPT $=\frac{\text { IPT inicial }}{}$

\section{O índice de eficiência do tratamento ${ }^{33}$}

A eficiência de um determinado protocolo de tratamento pode ser definida como a obtenção dos melhores resultados possíveis em um menor tempo de tratamento. Dessa forma, o índice de eficiência do tratamento é a razão entre a porcentagem de melhora e o tempo de tratamento em anos, podendo ser expresso pela seguinte fórmula matemática:

$\begin{gathered}\text { Eficiência do } \\ \text { tratamento }\end{gathered}=\frac{\begin{array}{c}\text { porcentagem de redução } \\ \text { do índice oclusal IPT }\end{array}}{\begin{array}{c}\text { tempo de tratamento } \\ \text { (em anos) }\end{array}}$

Diante do exposto acima, evidencia-se que a porcentagem de redução do índice oclusal e a eficiência do tratamento são grandezas diretamente proporcionais, ao passo que a eficiência e o tempo de tratamento são grandezas inversamente proporcionais. Sendo assim, o valor do índice de eficiência do tratamento aumenta na medida em que percentuais de melhora maiores são alcançados em menores tempos de tratamento.

\section{Telerradiografias em norma lateral}

Com a finalidade de avaliar as características cefalométricas iniciais dos grupos estudados, telerradiografias em norma lateral, obtidas ao início do tratamento, foram avaliadas. De acordo com as normas técnicas de obtenção radiográfica preconizadas pela Disciplina de Radiologia da Faculdade de Odontologia de Bauru da Universidade de São Paulo (FOB-USP), todas as imagens radiográficas foram obtidas com os lábios em repouso e na posição de máxima intercuspidação habitual, com o auxílio do cefalostato de Broadbent para uma 
padronização do posicionamento da cabeça.

Todas as telerradiografias da amostra foram realizadas em sete diferentes aparelhos, pois, para que houvesse um número suficiente de pacientes em cada grupo, necessitou-se selecionar a amostra a partir das documentações ortodônticas de todos os pacientes já tratados pela Disciplina de Ortodontia. Para aumentar a confiabilidade da caracterização cefalométrica desse estudo, foi realizada a determinação da magnificação de cada aparelho. Os diferentes tipos de aparelhos apresentaram porcentagens distintas de magnificação, que variaram entre $6 \%$ e $10,94 \%$.

\section{Elaboração do cefalograma}

Uma folha de papel "Ultraphan" de $0,07 \mathrm{~mm}$ de espessura e $17,5 \mathrm{~cm} \times 17,5 \mathrm{~cm}$ foi adaptada em cada telerradiografia. $\mathrm{O}$ traçado anatômico e a demarcação dos pontos cefalométricos dentoesqueléticos foram efetuados manualmente por um dos autores com lapiseira de $0,5 \mathrm{~mm}$, sobre um negatoscópio em sala escurecida. Em seguida, o traçado

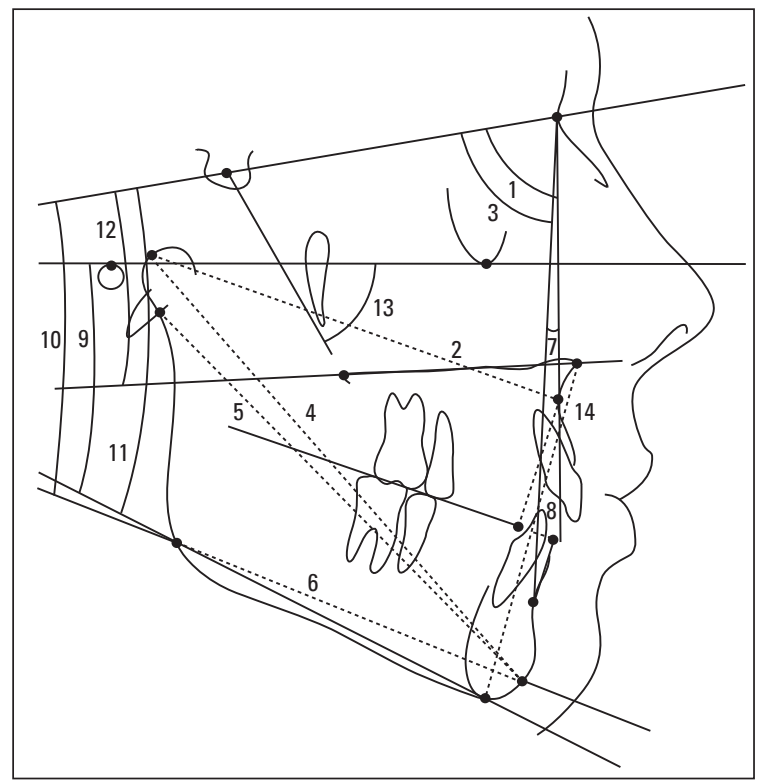

FIGURA 8 - Grandezas cefalométricas esqueléticas: (1) SNA; (2) Co-A; (3) SNB; (4) Co-Gn; (5) Ar-Gn; (6) GoGn; (7) ANB; (8) Wits; (9) FMA; (10) SN.GoGn; (11) SN.GoMe; (12) SN.PP; (13) eixo Y; (14) AFAI (altura facial ântero-inferior). anatômico e os pontos cefalométricos demarcados foram digitalizados com o auxílio de uma mesa digitalizadora Numonics AccuGrid XNT, modelo A30TL.F (Numonics Corp., Montgomeryville, Pennsylvania, EUA), interligada a um microcomputador. A mensuração das grandezas cefalométricas foi realizada pelo programa Dentofacial Planner 7.02 (Dentofacial Planner Software Inc., Toronto, Ontário, Canadá), efetuando-se por meio deste a correção da magnificação de cada imagem radiográfica. As grandezas angulares e lineares utilizadas no estudo encontram-se dispostas nas figuras 8 e 9.

\section{Análise estatística}

Erro do método

Para determinar a confiabilidade dos resultados, foram realizadas novamente, pelo mesmo pesquisador, todas as mensurações em trinta modelos de gesso e trinta telerradiografias em norma lateral, selecionadas aleatoriamente após um intervalo de três semanas. A aplicação da fórmula matemática

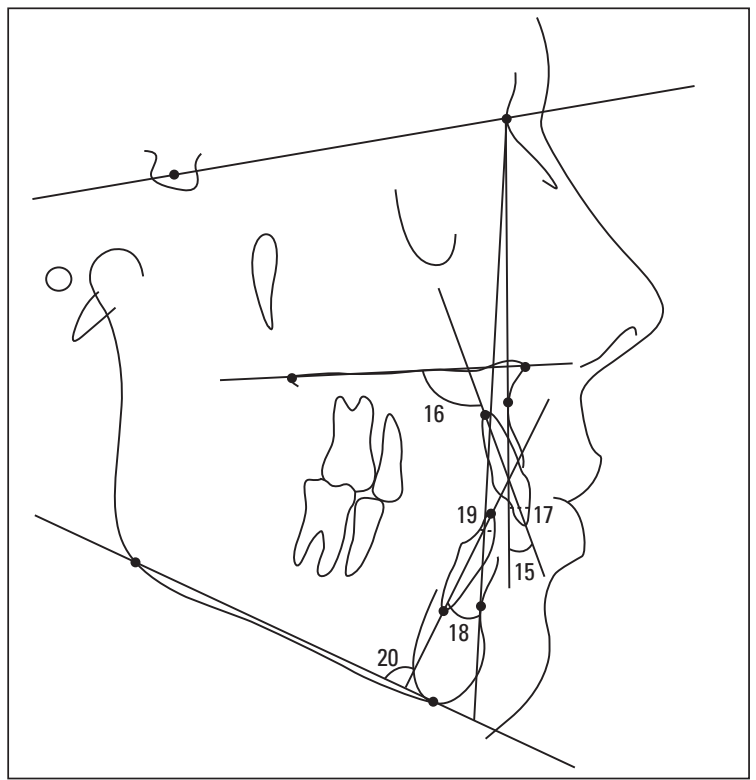

FIGURA 9 - Grandezas cefalométricas dentárias: (15) 1.NA; (16) 1.PP; (17) 1-NA; (18) 1.NB; (19) 1-NB; (20) IMPA. 
proposta por Dahlberg ${ }^{8}\left(\mathrm{Se}^{2}=\Sigma \mathrm{d}^{2} / 2 \mathrm{n}\right)$ permitiu estimar a ordem de grandeza dos erros casuais. A avaliação dos erros sistemáticos procedeu-se pela aplicação do teste $t$ dependente, conforme preconizado por Houston ${ }^{15}$.

\section{Comparação entre grupos e variáveis}

Para meio dessas comparações, foram realizados os seguintes testes estatísticos:

- Estatística descritiva: médias, desvios-padrão, valor máximo e valor mínimo das idades iniciais, finais e tempo total de tratamento nos dois grupos estudados.

- Teste $t$ independente: para avaliar a compatibilidade cefalométrica e comparar a idade inicial dos grupos 1 e 2 ao início do tratamento.

- Teste não paramétrico do qui-quadrado: para avaliar a compatibilidade dos dois grupos estudados quanto à severidade inicial da má oclusão no sentido ântero-posterior e a distribuição por gêneros.

- Teste $t$ independente: também foi utilizado para comparar a severidade da má oclusão no início (IPT inicial) e final do tratamento (IPT final), bem como as variáveis que quantificassem as alterações com o tratamento (IPTINICIAL - IPTFINAL), o percentual de melhora (\% IPT) e o grau de eficiência do tratamento (Eficiência IPT). Além disso, esse mesmo teste foi utilizado para comparar os dois grupos após compatibilizá-los quanto à idade inicial (com a exclusão dos 15 pacientes que apresentavam as maiores idades iniciais do grupo 1 e também dos 15 pacientes que apresentavam as menores idades iniciais do grupo 2) e ao tempo de tratamento (com a exclusão dos 19 pacientes que apresentavam os menores tempos de tratamento do grupo 1 e também os 19 pacientes que apresentavam os maiores tempos de tratamento do grupo 2). Esta compatibilização teve o objetivo de avaliar a influência destas variáveis (idade inicial e tempo de tratamento) nos resultados oclusais e na eficiência dos dois protocolos de tratamento que foram comparados.
- ANOVA a um critério: considerando que os pacientes do grupo 2 foram tratados com aparelhos distintos durante a fase ortopédica e a possibilidade deste aspecto influenciar os resultados do tratamento, o grupo 2 foi subdividido em subgrupos A (pacientes tratados na $1^{\text {a }}$ fase com o Ativador associado ao extrabucal) e B (pacientes tratados na $1^{\text {a }}$ fase com o Bionator de Balters). Dessa forma, as comparações entre o grupo 1 e subgrupos $2 \mathrm{~A}$ e 2B foi realizada por meio da análise de variância (ANOVA) a um critério.

- Teste de Tukey: para determinar entre quais grupos havia diferença estatisticamente significante quando detectada previamente pela ANOVA a um critério.

- Regressão linear múltipla: para avaliar qual variável (ANB inicial, gênero, aparelho utilizado, idade inicial, relação molar, IPT inicial e IPT final) possui uma maior influência no tempo de tratamento.

Todos os testes estatísticos foram realizados com o programa Statistica for Windows 6.0 (StatSoft Inc., Tulsa, Oklahoma, EUA) e os resultados foram considerados estatisticamente significantes para $\mathrm{p}<0,05$.

\section{RESULTADOS}

\section{Precisão da metodologia}

Apenas uma variável (Wits) apresentou erro sistemático $(\mathrm{p}<0,05)$ e a amplitude dos erros casuais variou de 0,31 (ANB) a 1,46 (IMPA) (Tab. 3).

Os dois grupos avaliados apresentaram compatibilidade ao início do tratamento, com exceção da idade inicial e das seguintes variáveis cefalométricas: Co-Gn, Ar-Gn, Go-Gn, AFAI, 1.NA e 1.PP (Tab. 4, 5).

Os valores apresentados demonstraram que resultados oclusais semelhantes são obtidos com os protocolos de tratamento em uma e duas fases. Além disso, o grupo 1 apresentou um grau de eficiência significantemente maior e um tempo de tratamento significantemente menor quando comparado ao grupo 2 (Tab. 6). 
Tabela 3 - Avaliação do erro intra-examinador. Resultados do teste t dependente (erro sistemático) e do erro casual.

\begin{tabular}{|c|c|c|c|c|c|c|}
\hline \multirow{2}{*}{ variáveis } & \multicolumn{2}{|c|}{$1^{\text {a }}$ medição $(n=30)$} & \multicolumn{2}{|c|}{$2^{\mathrm{a}}$ medição $(n=30)$} & \multirow{2}{*}{ Dahlberg (erro casual) } & \multirow{2}{*}{$\mathbf{p}$} \\
\hline & média & d.p. & média & d.p. & & \\
\hline \multicolumn{7}{|c|}{ modelos de gesso } \\
\hline IPT inicial & 7,67 & 1,59 & 7,61 & 1,66 & 0,3298 & 0,6383 \\
\hline IPT final & 1,31 & 1,19 & 1,28 & 1,22 & 0,1965 & 0,5648 \\
\hline \multicolumn{7}{|c|}{ telerradiografias em norma lateral } \\
\hline SNA $\left(^{\circ}\right)$ & 82,44 & 3,15 & 82,38 & 3,74 & 0,7523 & 0,9176 \\
\hline Co-A (mm) & 85,59 & 4,13 & 85,71 & 4,08 & 0,8195 & 0,8747 \\
\hline SNB $\left(^{\circ}\right)$ & 77,95 & 3,01 & 77,92 & 2,95 & 0,5528 & 0,9568 \\
\hline Co-Gn (mm) & 108,17 & 6,14 & 108,22 & 5,74 & 0,6220 & 0,9647 \\
\hline $\operatorname{Ar}-\mathrm{Gn}(\mathrm{mm})$ & 98,14 & 4,65 & 97,27 & 4,72 & 0,6764 & 0,3139 \\
\hline Go-Gn (mm) & 71,24 & 4,07 & 71,02 & 3,83 & 0,4395 & 0,7693 \\
\hline ANB $\left({ }^{\circ}\right)$ & 4,49 & 2,24 & 4,45 & 3,15 & 0,3175 & 0,9228 \\
\hline Wits (mm) & 4,72 & 2,92 & 5,89 & 3,11 & 1,2265 & $0,0002^{*}$ \\
\hline FMA $\left({ }^{\circ}\right)$ & 27,04 & 4,94 & 28,15 & 5,05 & 0,8091 & 0,2283 \\
\hline SN.GoGn $\left({ }^{\circ}\right)$ & 31,25 & 4,03 & 30,94 & 4,20 & 0,7088 & 0,6766 \\
\hline SN.GoMe $\left({ }^{\circ}\right)$ & 33,45 & 4,84 & 33,89 & 4,56 & 0,6649 & 0,6223 \\
\hline SN.PP $\left({ }^{\circ}\right)$ & 7,49 & 3,15 & 7,52 & 3,62 & 0,7554 & 0,9588 \\
\hline eixo facial $\left({ }^{\circ}\right)$ & 61,18 & 2,98 & 60,37 & 2,86 & 1,0345 & 0,1473 \\
\hline AFAl (mm) & 62,00 & 5,16 & 61,92 & 5,09 & 0,4582 & 0,9329 \\
\hline 1.NA $\left({ }^{\circ}\right)$ & 26,17 & 7,84 & 25,67 & 7,24 & 1,2205 & 0,7294 \\
\hline 1-NA (mm) & 5,10 & 2,77 & 5,08 & 2,53 & 0,3786 & 0,9687 \\
\hline 1.PP $\left({ }^{\circ}\right)$ & 115,42 & 7,52 & 116,40 & 7,96 & 1,2325 & 0,4811 \\
\hline 1.NB $\left({ }^{\circ}\right)$ & 26,35 & 6,44 & 26,74 & 6,72 & 1,0983 & 0,3341 \\
\hline 1-NB (mm) & 5,23 & 1,89 & 5,18 & 2,02 & 0,3205 & 0,8858 \\
\hline IMPA $\left({ }^{\circ}\right)$ & 96,55 & 7,43 & 96,87 & 7,15 & 1,4617 & 0,8152 \\
\hline
\end{tabular}

* Diferença estatisticamente significante $(p<0,05)$.

Tabela 4 - Avaliação da compatibilidade entre os grupos, considerando a idade inicial média (teste tindependente), a distribuição por gêneros (teste do qui-quadrado) e a severidade da relação ântero-posterior dos arcos dentários (teste do qui-quadrado).

\begin{tabular}{|c|c|c|c|}
\hline variáveis & $\begin{array}{l}\text { grupo } 1 \\
(\mathrm{n}=78)\end{array}$ & $\begin{array}{l}\text { grupo } 2 \\
(\mathrm{n}=61)\end{array}$ & $\mathbf{p}$ \\
\hline idade inicial - média (d.p.) & $12,51(1,28)$ & $11,21(1,21)$ & $0,0000 t^{*}$ \\
\hline gênero masculino & 41 & 30 & \multirow{2}{*}{$0,6921 q$} \\
\hline gênero feminino & 37 & 31 & \\
\hline relação molar 1/4 Classe II & 10 & 4 & \multirow{4}{*}{$0,1647 \mathrm{c}$} \\
\hline relação molar 1/2 Classe II & 21 & 14 & \\
\hline relação molar 3/4 Classe II & 26 & 16 & \\
\hline relação molar Classe II completa & 21 & 27 & \\
\hline
\end{tabular}

$\mathrm{t}=$ teste $\mathrm{t}$ independente; $\mathrm{q}=$ teste qui-quadrado.

* Diferença estatisticamente significante $(p<0,05)$. 
Tabela 5 - Resultados do teste t independente para avaliar a compatibilidade cefalométrica dos grupos 1 e 2 na fase inicial do tratamento.

\begin{tabular}{|c|c|c|c|c|c|}
\hline \multirow{2}{*}{ variáveis } & \multicolumn{2}{|c|}{ grupo $1(n=78)$} & \multicolumn{2}{|c|}{ grupo $2(n=61)$} & \multirow{2}{*}{$\mathbf{p}$} \\
\hline & média & d.p. & média & d.p. & \\
\hline \multicolumn{6}{|c|}{ componente maxilar } \\
\hline $\operatorname{SNA}\left({ }^{\circ}\right)$ & 81,69 & 3,93 & 81,37 & 3,05 & 0,5922 \\
\hline Co-A (mm) & 84,56 & 5,12 & 83,16 & 4,08 & 0,0830 \\
\hline \multicolumn{6}{|c|}{ componente mandibular } \\
\hline $\operatorname{SNB}\left({ }^{\circ}\right)$ & 76,51 & 3,47 & 75,55 & 2,74 & 0,0810 \\
\hline Co-Gn (mm) & 105,96 & 5,76 & 101,94 & 4,35 & $0,0000^{*}$ \\
\hline $\operatorname{Ar}-\mathrm{Gn}(\mathrm{mm})$ & 99,31 & 5,30 & 95,23 & 4,93 & $0,0000^{*}$ \\
\hline Go-Gn (mm) & 69,63 & 4,64 & 67,13 & 3,78 & $0,0008 *$ \\
\hline \multicolumn{6}{|c|}{ relação maxilomandibular } \\
\hline ANB $\left({ }^{\circ}\right)$ & 5,19 & 2,22 & 5,81 & 1,96 & 0,0851 \\
\hline Wits (mm) & 4,82 & 2,60 & 5,25 & 2,51 & 0,3281 \\
\hline \multicolumn{6}{|c|}{ padrão de crescimento } \\
\hline FMA $\left({ }^{\circ}\right)$ & 28,34 & 4,80 & 27,57 & 5,09 & 0,3580 \\
\hline SN.GoGn $\left({ }^{\circ}\right)$ & 33,88 & 5,69 & 33,72 & 5,13 & 0,8630 \\
\hline SN.GoMe $\left({ }^{\circ}\right)$ & 35,84 & 5,68 & 35,83 & 5,07 & 0,9906 \\
\hline SN.PP $\left({ }^{\circ}\right)$ & 7,52 & 3,61 & 7,58 & 2,70 & 0,9155 \\
\hline eixo facial $\left({ }^{\circ}\right)$ & 60,41 & 3,29 & 59,94 & 3,93 & 0,4379 \\
\hline AFAI (mm) & 61,74 & 5,11 & 59,89 & 4,29 & $0,0248 *$ \\
\hline \multicolumn{6}{|c|}{ componente dentoalveolar ântero-superior } \\
\hline 1.NA $\left(^{\circ}\right)$ & 25,59 & 8,76 & 28,87 & 6,92 & $0,0179 *$ \\
\hline 1-NA (mm) & 5,27 & 3,21 & 5,57 & 2,54 & 0,5438 \\
\hline 1.PP $\left({ }^{\circ}\right)$ & 114,80 & 7,37 & 117,82 & 7,18 & $0,0164^{*}$ \\
\hline \multicolumn{6}{|c|}{ componente dentoalveolar ântero-inferior } \\
\hline 1.NB $\left({ }^{\circ}\right)$ & 25,82 & 6,23 & 25,41 & 6,53 & 0,7070 \\
\hline $1-\mathrm{NB}(\mathrm{mm})$ & 4,61 & 2,35 & 4,06 & 2,23 & 0,1617 \\
\hline $\operatorname{IMPA}\left({ }^{\circ}\right)$ & 93,49 & 7,16 & 94,02 & 6,47 & 0,6491 \\
\hline
\end{tabular}

* Diferença estatisticamente significante $(p<0,05)$.

Tabela 6 - Resultados da comparação entre os grupos 1 e 2 nas variáveis estudadas por meio do teste t independente.

\begin{tabular}{|c|c|c|c|c|c|}
\hline \multirow{2}{*}{ variáveis } & \multicolumn{2}{|c|}{ grupo $1(n=78)$} & \multicolumn{2}{|c|}{ grupo $2(n=61)$} & \multirow[b]{2}{*}{$\mathbf{p}$} \\
\hline & média & d.p. & média & d.p. & \\
\hline idade final & 14,68 & 1,49 & 14,70 & 1,55 & 0,9160 \\
\hline tempo de tratamento (anos) & 2,17 & 0,66 & 3,49 & 1,56 & $0,0000^{*}$ \\
\hline IPT inicial & 7,86 & 2,36 & 8,13 & 2,11 & 0,4900 \\
\hline IPT final & 1,91 & 1,22 & 1,93 & 1,25 & 0,9161 \\
\hline IPTINICIAL - IPTFINAL & 5,95 & 2,53 & 6,19 & 2,44 & 0,5671 \\
\hline$\%$ IPT & 74,60 & 15,99 & 73,97 & 19,41 & 0,8362 \\
\hline eficiência IPT & 37,75 & 14,12 & 25,51 & 13,15 & $0,0000^{*}$ \\
\hline
\end{tabular}

* Diferença estatisticamente significante $(p<0,05)$. 
Após compatibilizar os grupos quanto à idade inicial, com a exclusão dos 15 pacientes que apresentavam as maiores idades iniciais do grupo 1 e também dos 15 pacientes que apresentavam as menores idades iniciais do grupo 2 , verificou-se que, ainda assim, o grupo 1 apresentou um grau de eficiência significantemente maior do que o grupo 2 (Tab. 7).

Após compatibilizar os grupos quanto ao tempo de tratamento, com a exclusão dos 19 pacientes que apresentavam os menores tempos de tratamento do grupo 1 e também os 19 pacientes que apresentavam os maiores tempos de tratamento do grupo 2, verificou-se uma semelhança entre os grupos 1 e 2 no que concerne à eficiência dos seus respectivos protocolos de tratamento (Tab. 8).

Considerando a possibilidade de aparelhos ortopédicos funcionais distintos propiciarem resultados oclusais também distintos e, dessa forma, atenuar ou acentuar as reais diferenças existentes

Tabela 7 - Resultados do teste $t$ independente aplicado às variáveis estudadas após compatibilizar os grupos 1 e 2 quanto à idade inicial.

\begin{tabular}{|c|c|c|c|c|c|}
\hline \multirow{2}{*}{ variáveis } & \multicolumn{2}{|c|}{ grupo $1(n=63)$} & \multicolumn{2}{|c|}{ grupo $2(n=46)$} & \multirow[b]{2}{*}{$\mathbf{p}$} \\
\hline & média & d.p. & média & d.p. & \\
\hline idade inicial & 12,07 & 0,94 & 11,73 & 0,86 & 0,0524 \\
\hline idade final & 14,22 & 1,19 & 14,82 & 1,51 & $0,0228 *$ \\
\hline tempo de tratamento (anos) & 2,15 & 0,65 & 3,09 & 1,35 & $0,0000^{*}$ \\
\hline IPT inicial & 7,53 & 1,86 & 8,11 & 2,10 & 0,1365 \\
\hline IPT final & 1,76 & 1,07 & 1,88 & 1,32 & 0,6158 \\
\hline IPTINICIAL - IPTFINAL & 5,77 & 1,96 & 6,23 & 2,31 & 0,2670 \\
\hline$\%$ IPT & 75,99 & 14,51 & 75,38 & 17,17 & 0,8422 \\
\hline eficiência IPT & 38,92 & 14,24 & 28,66 & 13,19 & $0,0002 *$ \\
\hline
\end{tabular}

* Diferença estatisticamente significante $(p<0,05)$.

Tabela 8 - Resultados do teste t independente aplicado às variáveis estudadas após compatibilizar os grupos 1 e 2 quanto ao tempo de tratamento.

\begin{tabular}{|c|c|c|c|c|c|}
\hline \multirow{2}{*}{ variáveis } & \multicolumn{2}{|c|}{ grupo $1(n=59)$} & \multicolumn{2}{|c|}{ grupo $2(n=42)$} & \multirow{2}{*}{$\mathbf{p}$} \\
\hline & média & d.p. & média & d.p. & \\
\hline tempo de tratamento (anos) & 2,37 & 0,64 & 2,59 & 0,65 & 0,0911 \\
\hline idade inicial & 12,61 & 1,24 & 11,51 & 1,16 & $0,0000^{*}$ \\
\hline idade final & 14,97 & 1,40 & 14,11 & 1,10 & $0,0011 *$ \\
\hline IPT inicial & 7,78 & 2,44 & 8,28 & 1,86 & 0,2646 \\
\hline IPT final & 2,01 & 1,15 & 1,95 & 1,29 & 0,7954 \\
\hline IPTINICIAL - IPTFINAL & 5,77 & 2,66 & 6,33 & 2,06 & 0,2516 \\
\hline$\%$ IPT & 72,51 & 15,92 & 75,49 & 15,85 & 0,3544 \\
\hline eficiência IPT & 32,91 & 11,24 & 31,23 & 11,66 & 0,4670 \\
\hline
\end{tabular}

* Diferença estatisticamente significante $(p<0,05)$. 
Tabela 9 - Resultados da análise de variância a um critério (ANOVA) e do teste de Tukey aplicados ao grupo 1 e subgrupos $2 \mathrm{~A}$ (Ativador + Aparelho fixo) e 2B (Bionator + Aparelho fixo).

\begin{tabular}{|c|c|c|c|c|}
\hline variáveis & $\begin{array}{l}\text { grupo } 1 \text { ( } \mathrm{n}=78 \text { ) } \\
\text { média (d.p.) }\end{array}$ & $\begin{array}{c}\text { subgrupo } 2 A(n=30) \\
\text { média (d.p.) }\end{array}$ & $\begin{array}{c}\text { subgrupo 2B (n=31) } \\
\text { média (d.p.) }\end{array}$ & ANOVA p \\
\hline idade inicial & $12,51(1,28)^{\mathrm{A}}$ & $11,08(1,25)^{\mathrm{B}}$ & $11,34(1,18)^{\mathrm{B}}$ & $0,0000^{*}$ \\
\hline idade final & $14,68(1,49)^{\mathrm{A}}$ & $13,89(1,18)^{\mathrm{B}}$ & $15,49(1,48)^{C}$ & $0,0001^{*}$ \\
\hline tempo de tratamento (anos) & $2,17(0,66)^{\mathrm{A}}$ & $2,81(1,10)^{\mathrm{B}}$ & $4,15(1,67)^{\mathrm{C}}$ & $0,0000^{*}$ \\
\hline IPT inicial & $7,86(2,36)^{A}$ & $7,74(1,78)^{\mathrm{A}}$ & $8,50(2,36)^{A}$ & 0,3370 \\
\hline IPT final & $1,91(1,22)^{A}$ & $2,16(1,35)^{\mathrm{A}}$ & $1,72(1,12)^{A}$ & 0,3750 \\
\hline IPTINICIAL - IPTFINAL & $5,95(2,53)^{\mathrm{A}}$ & $5,59(2,09)^{\mathrm{A}}$ & $6,78(2,64)^{A}$ & 0,1459 \\
\hline$\%$ IPT & $74,60(15,99)^{A}$ & $70,27(21,64)^{A}$ & $77,56(16,54)^{\mathrm{A}}$ & 0,2635 \\
\hline eficiência IPT & $37,75(14,12)^{A}$ & $28,21(11,88)^{\mathrm{B}}$ & $22,90(13,96)^{\mathrm{B}}$ & $0,0000^{*}$ \\
\hline
\end{tabular}

* Diferença estatisticamente significante $(p<0,05)$. Letras diferentes representam diferença estatisticamente significante no teste de Tukey.

Tabela 10 - Resultados da análise de regressão linear múltipla considerando o tempo de tratamento como variável dependente.

\begin{tabular}{|c|c|c|c|c|}
\hline variáveis & $\begin{array}{l}\text { coeficiente parcial de } \\
\text { regressão }\end{array}$ & erro padrão & $\mathbf{t}$ & $\mathbf{p}$ \\
\hline ANB inicial & 0,103 & 0,071 & 1,453 & 0,1487 \\
\hline gênero & 0,035 & 0,069 & 0,508 & 0,6125 \\
\hline aparelho utilizado & 0,493 & 0,076 & 6,479 & $0,0000^{*}$ \\
\hline idade inicial & $-0,183$ & 0,078 & $-2,350$ & $0,0203^{*}$ \\
\hline relação molar & 0,080 & 0,070 & 1,138 & 0,2571 \\
\hline IPT inicial & 0,010 & 0,071 & 0,139 & 0,8895 \\
\hline IPT final & 0,046 & 0,070 & 0,662 & 0,5090 \\
\hline
\end{tabular}

* Diferença estatisticamente significante $(p<0,05) \cdot R^{2}=0,4000$.

entre os dois protocolos de tratamento avaliados, decidiu-se dividir o grupo 2 em subgrupos 2A (composto de 30 pacientes que foram tratados na fase ortopédica com o Ativador associado ao aparelho extrabucal) e 2B (composto de 31 pacientes tratados com o Bionator de Balters na primeira fase do tratamento) de acordo com o aparelho funcional utilizado como forma de correção da má oclusão de Classe II na primeira fase do tratamento (Tab. 9). Diante dos resultados dispostos na tabela 9, verifica-se que o subgrupo $2 \mathrm{~B}$ apresenta um tempo de tratamento significantemente maior do que o grupo 1 e o subgrupo 2A, fato este que contribuiu para que a idade final do subgrupo $2 \mathrm{~B}$ também fosse significantemente maior do que o grupo 1 e o subgrupo 2A (Tab. 9). As outras variáveis analisadas tiveram um comportamento semelhante ao encontrado na comparação entre os grupos 1 e 2 (Tab. 6): idade inicial significantemente maior para o grupo 1 , quando comparado aos subgrupos 2A e 2B, que não exibiram diferenças entre si; variáveis diretamente relacionadas aos índices oclusais sem apresentar diferenças na comparação entre o grupo 1 e subgrupos $2 \mathrm{~A}$ e $2 \mathrm{~B}$; e um grau 
de eficiência significantemente maior para o grupo 1 quando comparado aos subgrupos $2 \mathrm{~A}$ e $2 \mathrm{~B}$, que não exibiram diferenças entre si.

A análise de regressão linear múltipla - considerando o tempo de tratamento como a variável dependente - identificou apenas duas variáveis que influenciam significantemente o tempo de tratamento: (1) aparelho utilizado e (2) idade inicial (Tab. 10).

\section{DISCUSSÃO}

\section{Compatibilidade dos grupos estudados}

Durante a seleção da amostra, a severidade da relação ântero-posterior dos arcos dentários, ao início do tratamento, não se constituiu em um critério de inclusão e/ou exclusão dos pacientes. No entanto, ao avaliar a compatibilidade entre os grupos considerando essa discrepância ânteroposterior dos arcos, verificou-se que os dois grupos eram semelhantes (Tab. 4). Essa similaridade entre os grupos é uma condição sine qua non para comparar os resultados oclusais e a eficiência dos dois protocolos de tratamento considerados nesse estudo, uma vez que a efetividade da correção da má oclusão de Classe II está diretamente relacionada com a severidade inicial da discrepância ânteroposterior ${ }^{21}$.

A incompatibilidade dos grupos quanto à idade inicial poderia influenciar os resultados desse trabalho, no sentido de favorecer a obtenção de melhores resultados oclusais no grupo tratado em duas fases, visto que esse grupo apresentava uma idade média menor ao início do tratamento e, por conseguinte, um maior período de crescimento, favorecendo a correção da má oclusão de Classe II ${ }^{19}$ (Tab. 4). De uma certa forma, essa diferença significante entre as idades iniciais dos dois grupos estudados já era esperada, uma vez que o sucesso de um tratamento ortopédico está na dependência do aproveitamento do surto de crescimento pubescente do paciente e que, conseqüentemente, implica em iniciar o tratamento em uma fase mais precoce ${ }^{2,20}$. Além disso, trabalhos existentes na literatura com propostas semelhantes à do presente estudo já evidenciaram essa diferença entre as idades iniciais médias dos grupos que foram comparados 22,33 . No entanto, com o intuito de verificar a influência dessa variável nos resultados aqui apresentados, realizou-se uma segunda comparação das variáveis estudadas após compatibilizar os grupos quanto à idade inicial (Tab. 7).

Considerando a existência de diferenças importantes entre os gêneros masculino e feminino, no que diz respeito às respostas biológicas a um determinado protocolo de tratamento ortodôntico ${ }^{3}$, a compatibilidade entre os grupos - no que se refere à distribuição quanto ao gênero - é fundamental para que essa variável não tenha influência nos resultados apresentados (Tab. 4). Além disso, alguns autores têm relatado que há diferença entre os gêneros quando considera-se o nível de colaboração conseguida durante o tratamento, beneficiando ou comprometendo as metas do tratamento ${ }^{6,32}$. Ao admitirmos que o potencial de crescimento presente é um fator importante na correção da Classe II e que o sucesso dos diversos métodos de correção dessa má oclusão dependem, em algum grau, da cooperação do paciente $e^{4,17,18,19}$, a compatibilidade dos grupos quanto à distribuição por gêneros foi de grande importância (Tab. 4).

Para a avaliação dos resultados oclusais e da eficiência dos protocolos de tratamento em uma e duas fases, procurou-se realizar uma comparação entre grupos com características morfológicas semelhantes. Na tentativa de se obter resultados mais confiáveis e menos tendenciosos, estudos recentes têm demonstrado a preocupação de avaliar o grau de compatibilidade cefalométrica entre os grupos que estão sendo comparados ${ }^{22,34}$. Além disso, uma caracterização cefalométrica dos grupos ao início do tratamento permite avaliar a participação dos distintos componentes esqueléticos e dentários associados à má oclusão, bem como realizar uma comparação dos grupos no que se refere ao padrão de crescimento craniofacial predominante. 
De uma maneira geral, os grupos apresentaram uma compatibilidade cefalométrica satisfatória ao início do tratamento, sendo que, das vinte variáveis cefalométricas avaliadas, seis apresentaram diferenças estatisticamente significantes (Tab. 5). As diferenças entre os grupos concentraram-se principalmente nas variáveis que avaliam o comprimento mandibular (Co-Gn, Ar-Gn e Go-Gn), na altura facial ântero-inferior (AFAI) e na inclinação dos incisivos superiores (1.NA e 1.PP). Uma provável explicação para as diferenças encontradas nas dimensões esqueléticas é a idade inicial significantemente maior no grupo 1, quando comparado ao grupo 2, como também foi evidenciado em um estudo semelhante ${ }^{22}$ (Tab. 5).

Uma maior inclinação para vestibular dos incisivos superiores no grupo 2 foi, provavelmente, conseqüência de uma maior quantidade de indivíduos com Classe II completa neste grupo (Tab. 4, 5).

\section{Resultados da comparação entre os grupos}

Os resultados da comparação intergrupos evidenciaram que o grupo 1 apresentou um grau de eficiência significantemente maior e um tempo de tratamento significantemente menor, quando comparado ao grupo 2 (Tab. 6). Um tempo de tratamento significantemente maior nos pacientes que receberam o protocolo de tratamento em duas fases adveio do fato de que os pacientes do grupo 2 iniciaram o tratamento da Classe II em uma idade mais precoce e finalizaram o tratamento ortodôntico em uma idade mais avançada (Tab. 1). Considerando a comparação entre os grupos, no que diz respeito à variável referente ao resultado oclusal, constatou-se uma similaridade no que concerne à relação oclusal final (IPTFINAL), às alterações proporcionadas pelos protocolos de tratamento estudados (IPTINICIAL - IPTFINAL) e ao percentual de melhora do índice oclusal (\% IPT) (Tab. 6).

Os resultados obtidos neste trabalho confirmam os achados de King et al. ${ }^{21}$, que demonstraram que resultados oclusais semelhantes são obtidos ao final do tratamento, independente do protocolo de tratamento adotado.

Objetivando avaliar a influência da incompatibilidade dos grupos quanto à idade inicial, nos resultados dispostos na tabela 6 , decidiu-se compatibilizar os grupos quanto à idade inicial (Tab. 7). Após obter-se esta compatibilidade entre os dois grupos na variável idade inicial - com a exclusão dos 15 pacientes que apresentavam as maiores idades iniciais do grupo 1 e também dos 15 pacientes que apresentavam as menores idades iniciais do grupo 2 -, verificou-se que, ainda assim, o grupo 1 apresentou um grau de eficiência significantemente maior do que o grupo 2 (Tab. 7). Diante dos resultados apresentados na tabela 7 , pode-se concluir que a variável idade inicial apresenta pouca ou nenhuma influência nos resultados oclusais alcançados em ambos os grupos, bem como na eficiência de ambos os protocolos de tratamento.

Após uma minuciosa análise da tabela 6, podese especular que a maior eficiência do protocolo de tratamento em uma fase foi severamente influenciada pelo menor tempo de tratamento do grupo 1. Considerando-se que as variáveis tempo de tratamento e grau de eficiência são grandezas inversamente proporcionais e que o percentual de melhora dos índices oclusais utilizados foi semelhantes em ambos os grupos, fica mais evidente a influência do tempo de tratamento no grau de eficiência de ambos os protocolos de tratamento. A fim de constatarmos de forma transparente e inquestionável essa influência, decidiu-se compatibilizar os grupos quanto ao tempo de tratamento (Tab. 8). Após obtermos esta compatibilidade entre os dois grupos, na variável tempo de tratamento - com a exclusão dos 19 pacientes que apresentavam os menores tempos de tratamento do grupo 1 e também os 19 pacientes que apresentavam os maiores tempos de tratamento do grupo 2 - verificou-se uma semelhança entre os grupos 1 e 2 no que concerne à eficiência dos seus respectivos protocolos de tratamento (Tab. 8). Assim sendo, 
fica óbvio que o menor tempo de tratamento do grupo 1 foi também responsável pela maior eficiência desse grupo.

Admitindo-se que o Ativador e o Bionator são aparelhos que promovem alterações semelhantes em intervalos de tempo também semelhantes ${ }^{5,13}$, decidiu-se investigar a explicação para a existência de diferença estatisticamente significante entre os subgrupos $2 \mathrm{~A}$ e $2 \mathrm{~B}$ na variável tempo de tratamento (Tab. 9). Após pormenorizar todas as etapas do tratamento em duas fases de ambos os grupos, verificou-se que foi no tempo de tratamento ortopédico que o subgrupo 2A mostrou-se significantemente menor. Uma das possiveis explicações para esse fato é que a associação do aparetho extrabucal (IHG) ao Ativador faz com que as alterações esqueléticas e dentoalveolares ocorram em um intervalo de tempo menor, quando comparado à utilização de um aparelho funcional isoladamente - como aconteceu no subgrupo 2B. A justificativa para esse aspecto é que as ações do extrabucal com o Ativador não apenas são complementares, mas também seus respectivos efeitos aumentam de tal maneira que passam a representar um importante efeito terapêutico ${ }^{7,25}$. A força do aparelho extrabucal associado ao Ativador, além de exercer um efeito restritivo no deslocamento da porção anterior da maxila, propicia um maior controle do tratamento no sentido vertical, impedindo uma rotação horária da maxila e, conseqüentemente, da mandíbula, cuja rotação seria prejudicial no tratamento da Classe II $^{7,25}$.

Assim sendo, fica evidente que a associação do aparelho extrabucal com os aparelhos funcionais apresenta uma maior eficiência clínica em corrigir a má oclusão de Classe II, divisão 1, controlando alguns efeitos desfavoráveis provocados pelo uso isolado dos aparelhos funcionais. No entanto, a idéia de que essa terapia potencializa os efeitos relacionados ao crescimento mandibular parece equivocada. Ela apenas aumenta o crescimento diferencial da mandíbula em relação à maxila, devido à restrição significante do crescimento maxilar, fato esse que não reduz a importância da Ortopedia Funcional. A Ortopedia deve ser encarada como uma conduta clínica válida, pois essa terapêutica é considerada mais fisiológica do que o tratamento convencional com aparelhos fixos, propiciando uma redução significativa da discrepância esquelética e uma melhor harmonia das características faciais e dentárias.

O tempo total de tratamento foi a variável responsável por conferir uma maior eficiência ao protocolo de tratamento em uma fase, uma vez que resultados oclusais semelhantes são obtidos com os dois protocolos de tratamento, porém esses resultados são alcançados em tempos consideravelmente maiores no grupo 2 (Tab. 6). Além disso, ao compatibilizarmos os grupos 1 e 2 quanto ao tempo total de tratamento, verificou-se também uma compatibilidade quanto ao grau de eficiência (Tab. 8). Esses resultados estão em concordância com outros autores que demonstraram que o tratamento da Classe II em duas fases prolonga o tempo total de tratamento ${ }^{16,22,28}$.

Diante da importância do tempo de tratamento nos objetivos desse trabalho, decidiu-se identificar as variáveis que mais influenciaram a duração do tratamento da Classe II, divisão 1 (Tab. 10). Assim sendo, uma análise de regressão linear múltipla foi aplicada a variáveis relacionadas aos modelos (relação molar, IPT inicial e IPT final), a uma variável cefalométrica (ANB inicial), às características individuais do paciente (gênero, idade inicial) e também a uma variável diretamente relacionada à forma de correção da Classe II (aparelho utilizado) (Tab. 10). Diante dos resultados da análise de regressão, verificou-se que somente o aparelho utilizado e a idade inicial estão significantemente relacionados com o tempo de tratamento (Tab. 10). As demais variáveis apresentaram um valor de $\mathrm{p}$ não significante estatisticamente $(\mathrm{p}>$ $0,05)$, mostrando que não interferem na duração do tratamento da má oclusão de Classe II. As sete variáveis que foram incluídas no modelo de regressão explicam $40 \%$ da variação do tempo de 
tratamento nas más oclusões de Classe II, divisão 1, independente do protocolo de tratamento (Tab. 10). A forma de correção da Classe II (aparelho utilizado) é a variável que mais explica a variação do tempo de tratamento, uma vez que possui o maior coeficiente parcial de regressão dentre todas as variáveis investigadas. A idade inicial também tem uma influência direta na variação do tempo de tratamento e o sinal negativo do coeficiente de regressão mostra que quanto menor a idade do paciente maior será o tempo de tratamento, independente do protocolo adotado (Tab. 10). O resultado deste modelo de regressão foi semelhante ao obtido por Popowich et al..$^{27}$, que demonstraram que o aparelho utilizado e a idade inicial possuem uma influência significante no tempo de tratamento das más oclusões de Classe II.

\section{CONCLUSÕES}

Baseado nos resultados obtidos e na metodologia empregada, verificou-se que resultados oclusais semelhantes são obtidos com os dois protocolos de tratamento avaliados, porém um menor tempo de tratamento foi responsável por atestar uma maior eficiência ao protocolo de tratamento em uma fase. Portanto, resultados oclusais semelhantes - obtidos em tempos de tratamento consideravelmente menores - comprovam uma maior eficiência do protocolo de tratamento em uma fase, em comparação com o tratamento em duas fases.

\title{
Efficiency of 1-phase and 2-phase treatment protocols in Class II, division 1 malocclusions
}

\begin{abstract}
Aim: The purpose of this study was to compare the occlusal outcomes and the efficiency of 1-phase and 2-phase treatment protocols in Class II, division 1 malocclusions. Treatment efficiency was defined as a better dentoalveolar result in a shorter treatment time. Methods: Class II, division 1 subjects $(n=139)$ were divided into two groups in agreement with the treatment protocol adopted for Class II correction. Group 1 comprised 78 patients treated with 1-phase treatment protocol (single-stage group) at initial and final mean ages of $12.51( \pm 1.28)$ and 14.68 ( \pm 1.49) years. Group 2 comprised 61 patients treated with 2 -phase treatment protocol (two-stage group) at initial and final mean ages of $11.21( \pm 1.21)$ and $14.70( \pm 1.55)$ years. Lateral cephalometric radiographs were taken at the pretreatment stage to evaluate morphological differences among treatment groups. Evaluations were performed on the initial and final study models of the patients using treatment priority index (TPI). Chi-square tests were used to compare the two groups regarding initial molar Class and gender distribution. Variables regarding occlusal results were compared using independent t-tests. Finally, a multiple linear regression analysis was completed, with total treatment time as the dependent variable to identify clinical factors that predict treatment length for patients with Class II malocclusions. Results: Results demonstrated that similar occlusal outcomes are obtained between 1-phase and 2-phase treatment protocols, but the duration of treatment was significantly smaller in the 1-phase treatment protocol group. Conclusion: Based on the results of this investigation, it can be concluded that treatment of Class II, division 1 malocclusions is more efficient with the 1-phase treatment protocol.
\end{abstract}

Key words: Angle Class II malocclusion. Treatment outcome. Activator appliances. 


\section{REFERÊNCIAS}

1. ASCHER, F. The Bionator. In: GRABER, T. M.; NEUMANN, B. Removable orthodontic appliances. 2 nd ed. Philadelphia: Saunders, 1984. cap. 9, p. 229-246.

2. BASS, N. M. Orthopedic coordination of dentofacial development in skeletal Class II malocclusion in conjunction with Edgewise therapy. Part I. Am. J. Orthod., St. Louis, v. 84, no. 5, p. 361-383, Nov. 1983.

3. BISHARA, S. E. et al. Longitudinal changes in standing height and mandibular parameters between the ages of 8 and 17 years. Am. J. Orthod., St. Louis, v. 80, no. 2, p. 115-135, Aug 1981.

4. BISHARA, S. E.; ZIAJA, R. R. Functional appliances: a review. Am. J. Orthod. Dentofacial Orthop. St. Louis, v. 95, no. 3 p. 250-258, Mar. 1989.

5. CRUZ, K. S. et al. Efeitos dos aparelhos funcionais na correção da má oclusão de Classe II. Rev. Dental Press Ortodon. Ortop. Facial, Maringá, v. 5, n. 4, p. 43-52, jul./ago. 2000.

6. CUCALON, A.; SMITH, R. J. Relationship between compliance by adolescent orthodontic patients and performance on psychological tests. Angle Orthod., Appleton, v. 60, no. 2, p. 107-114, Apr. 1990

7. CURA, N. et al. Orthodontic and orthopedic effects of Activator, Activator-HG combination, and Bass appliances: a comparative study. Am. J. Orthod. Dentofacial Orthop., St. Louis, v. 110, no. 1, p. 36-45, July 1996.

8. DAHLBERG, G. Statistical methods for medical and biological students. New York: Interscience, 1940.

9. DUGONI, S. A.; LEE, J. S. Mixed dentition case report. Am. J. Orthod. Dentofacial Orthop., St. Louis, v. 107, no. 3, p. 239244, Mar. 1995.

10. FIROUZ, M.; ZERNIK, J.; NANDA, R. Dental and orthopedic effects of high-pull headgear in treatment of Class II, division 1 malocclusion. Am. J. Orthod. Dentofacial Orthop., St. Louis, v. 102, no. 3, p. 197-205, Sept. 1992.

11. FREITAS, M. R. et al. Prevalência das más oclusões em pacientes inscritos para tratamento ortodôntico na Faculdade de Odontologia de Bauru-USP. Rev. Fac. Odontol., Bauru, v. 10, n. 3, p. 164-169, jul./set. 2002.

12. GIANELLY, A. A.; ARENA, S. A.; BERNSTEIN, L. A comparison of Class II treatment changes noted with the light wire, Edgewise, and Fränkel appliances. Am. J. Orthod., St. Louis, v. 86 , no. 4, p. 269-276, Oct. 1984.

13. GRABER, T. M.; NEUMANN, B. Removable orthodontic appliances. 2nd. Philadelphia: Saunders, 1984.

14. GRAINGER, R. M. Orthodontic treatment priority index. Washington, DC: Dept. of Health Education and Welfare Public Health Service, 1967. Public Health Service Publication, no. 1000.

15. HOUSTON, W. J. The analysis of errors in orthodontic measurements. Am. J. Orthod., St. Louis, v. 83, no. 5, p. $382-$ 390, May 1983.

16. HSIEH, T.; PINSKAYA, Y.; ROBERTS, W. E. Assessment of orthodontic treatment outcomes: early treatment versus late treatment. Angle Orthod., Appleton, v. 75, no. 2, p. 158-166, Mar. 2005.

17. JANSON, G. et al. Class II treatment success rate in 2- and 4- premolar extraction protocols. Am. J. Orthod. Dentofacial Orthop., St. Louis, v. 125, no. 4, p. 472-479, Apr. 2004.

18. JANSON, G. et al. Occlusal changes of Class II malocclusion treatment between Fränkel and the eruption guidance appliances. Angle Orthod., Appleton, v. 74, no. 4, p. 521-525, Aug. 2004.

19. JANSON, G. R. P. et al. Avaliação do grau de colaboração e aceitação dos pacientes na utilização de dois diferentes tipos de aparelhos removíveis. Rev. Dental Press Ortodon. Ortop. Facial, Maringá, v. 8, n. 2, p. 31-40, mar./abr. 2003.

20. JANSON, G. R. P. et al. Class II treatment effects of the Fränkel appliance. Eur. J. Orthod., Oxford, v. 25, no. 3, p. 301-319, June 2003.
21. KING, G. J. et al. Comparison of peer assessment ratings (PAR) from 1-phase and 2-phase treatment protocols for Class II malocclusions. Am. J. Orthod. Dentofacial Orthop., St. Louis, v. 123, no. 5, p. 489-496, May 2003.

22. LIVIERATOS, F. A.; JOHNSTON JR., L. E. A comparison of one-stage and two-stage nonextraction alternatives in matched Class II samples. Am. J. Orthod. Dentofacial Orthop., St. Louis, v. 108, no. 2, p. 118-131, Aug. 1995.

23. McCULLOUGH, S. Angle Class II, division 1 malocclusion treated without premolar extraction. Am. J. Orthod. Dentofacial Orthop., St. Louis, v. 106, no. 3, p. 317-321, Sept. 1994.

24. NELSON, B.; HANSEN, K.; HÄGG, U. Class II correction in patients treated with Class II elastics and with fixed functional appliances: a comparative study. Am. J. Orthod. Dentofacial Orthop., St. Louis, v. 118, no. 2, p. 142-149, Aug. 2000.

25. ÖZTÜRK, Y.; TANKÜTER, N. Class II: a comparison of activator and activator headgear combination appliances. Eur. J. Orthod., Oxford, v. 16, no. 2, p. 149-157, Apr. 1994.

26. PARK, C. O.; CHO, K. H. Nonextraction treatment of a Class II division 1 malocclusion. Am. J. Orthod. Dentofacial Orthop. St. Louis, v. 109, no. 3, p. 227-333, Mar. 1996.

27. POPOWICH, K. et al. Predictors for Class II treatment duration. Am. J. Orthod. Dentofacial Orthop., St. Louis, v. 127, no. 3, p. 293-300, Mar. 2005

28. PROFFIT, W. R.; TULLOCH, J. F. Preadolescent Class II problems: treat now or wait? Am. J. Orthod. Dentofacial Orthop., St. Louis, v. 121, no. 6, p. 560-562, June 2002

29. RIGHELLIS, E. G. Treatment effects of Fränkel, activator and extraoral traction appliances. Angle Orthod., Appleton, v. 53, no. 2, p. 107-121, Apr. 1983

30. RUDZKI-JANSON, I.; NOACHTAR, R. Functional appliance therapy with the Bionator. Semin. Orthod., Philadelphia, v. 4, no. 1, p. 33-45, Mar. 1998.

31. SCHIAVON GANDINI, M. R. et al. Effects of cervical headgear and edgewise appliances on growing patients. Am. J. Orthod. Dentofacial Orthop., St. Louis, v. 119, no. 5, p. 531-538, May 2001. Discussion 538-539.

32. STARNBACH, H. K.; KAPLAN, A. Profile of an excellent orthodontic patient. Angle Orthod., Appleton, v. 45, no. 2, p. 141-145, Apr. 1975.

33. VON BREMEN, J.; PANCHERZ, H. Efficiency of early and late Class II division 1 treatment. Am. J. Orthod. Dentofacial Orthop., St. Louis, v. 121, no. 1, p. 31-37, Jan. 2002.

34. WHEELER, T. T. et al. Effectiveness of early treatment of Class II malocclusion. Am. J. Orthod. Dentofacial Orthop., St. Louis, v. 121, no. 1, p. 9-17, Jan. 2002.

\section{Endereço para correspondência}

Rodrigo Hermont Cançado

Unidade de Ensino Superior Ingá (UNINGÁ)

Av. Colombo, $9.727 \mathrm{Km} 130$

CEP: 87.070- 810 - Maringá/PR

E-mail: rohercan@uol.com.br 